\title{
Elastic Stresses of Rotating Transversely Isotropic Fiber Reinforced Composite Cylinders
}

\author{
Ömer Can Farukoğlu ${ }^{a^{*}}$, Ihsan Korkut ${ }^{b}$ \\ ${ }^{a, b}$ Department of Manufacturing Engineering, Gazi University, Ankara, Turkey. \\ E-mail address: omerfarukoglu08@gmail.com ${ }^{\text {a }}$, ikorkut@gazi.edu.tr ${ }^{\text {b }}$
}

ORCID numbers of author: 0000-0003-3244-8355 ${ }^{a}, 0000-0002-5001-4449^{b}$

Received date: 02.10 .2020

Accepted date: 24.12 .2020

\begin{abstract}
In this study, rotating long thick-walled fiber reinforced composite cylinders with closed ends are investigated within the elastic limits by using analytical methods. Hoffman yield criterion is employed to the elastic problem to find limit angular velocities. Composite bodies of the cylinders are consisting of isotropic matrix and transversely isotropic fibers which are unidirectionally aligned in the circumferential direction. Alterations on the elastic stress and displacement fields are examined by taking various fiber volume fraction and wall thickness values. Obtained results emphasize that both parameters highly influence the distributions of stress, displacement, and commencement of the yielding.
\end{abstract}

Keywords: Rotating cylinders; composite cylinders; stress analysis; analytical methods

\section{Introduction}

In many engineering applications, cylindrical components such as disks, cylinders, and rods are often utilized. Hence, it is highly crucial to forecast stress and displacement distributions in such geometries. According to the developments in material science and the needs in engineering, axisymmetric components have started to be produced from different materials. Fiber reinforced composites have become exceedingly popular among scientists and engineers due to the advantageous material properties. In this regard, stress analyses of axisymmetric components, which are made of different materials, under various loading conditions can be found extensively in the literature. Stress analyses of rotating functionally graded material (fgm) disks have been broadly examined by the use of analytical and numerical methods [1-5]. Deformations and stresses of pressurized fgm cylinders and tubes can be found in the publications as well [6-10]. Similar studies are also carried out for orthotropic cylindrical structures. Rotating orthotropic disks have been the subject of various engineering studies [1114]. Likewise, solutions have been proposed to orthotropic cylinder investigations with $[15,16]$ or without $[17,18]$ the influence of thermal stresses. Several studies focus on composite disks as well. Stress and displacement fields of solid, annular, and variable thickness rotating fiber reinforced disks are available [19-21]. On the other hand, the number of researches for the fiber reinforced disks and cylinders is lower than the same geometries made of functionally graded 
or orthotropic materials. Thus, this study is aiming to give more insight into fiber reinforced cylinders by employing analytical methods.

In this research, rotating long thick-walled composite cylinders with fixed ends are studied in the framework of elasticity. The composite material of the cylinders consisting of isotropic matrix and transversely isotropic fibers. The alignment of the fibers is taken unidirectionally through the circumferential direction. Thus, the circumferential direction becomes the longitudinal $(L)$ direction, and the remaining radial and axial directions of the cylinders turn out to be the transverse $(T)$ directions. In Fig. 1 (a), these directions and the composite cylinders are visualized. In Fig. 1 (b), fiber reinforced composite material is demonstrated with the material coordinate system. Correspondingly, lower case $l$ and $t$ point to the longitudinal (fiber) and transverse directions of the composite material, where $m$ and $f$ denote matrix and fibers as well. In the elastic limit calculations, Hoffman yield criterion [22] is exploited to obtain elastic limit angular velocity and commencement of the yielding through the thickness of the cylinders.

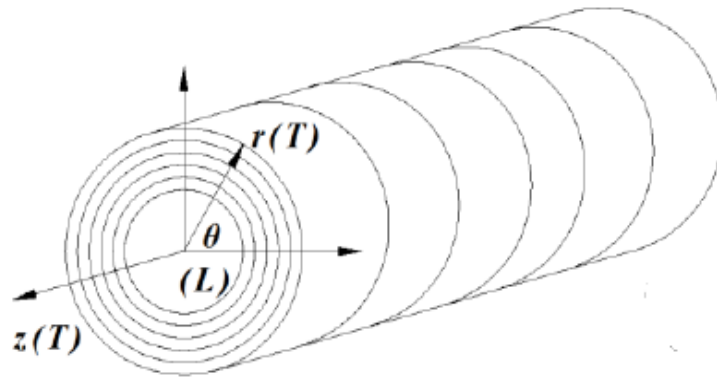

(a)

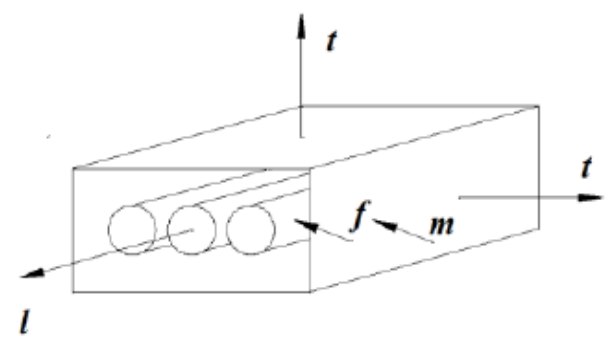

(b)

Fig.1. (a) Rotating cylinders with central hole, (b) Composite material

\section{Mechanical Property Calculations}

Several models can be supplied from the literature to calculate the mechanical properties of the fiber reinforced composites. In the present work, Chamis method [23,24] is utilized due to its usefulness and simple implementation. $V_{m}$ and $V_{f}$ denote the volume fraction of the matrix and fibers in Eq.(1).

$$
V_{m}=1-V_{f}
$$

Elastic modulus of the composite material in the longitudinal $\left(E_{L}\right)$ and transverse $\left(E_{T}\right)$ directions are calculated via Eqs.(2)-(3)

$$
\begin{gathered}
E_{L}=V_{f} E_{l f}+V_{m} E_{m} \\
E_{T}=\frac{E_{m}}{1-\sqrt{V_{f}}\left(1-\frac{E_{m}}{E_{t f}}\right)}
\end{gathered}
$$

where $E_{l f}$ and $E_{t f}$ express elastic modulus of the transversely isotropic fibers in the longitudinal and transverse directions. $E_{m}$ is the elastic modulus of the matrix. Followingly, Poisson's ratios of the composite material in different directions $\left(v_{L T}, v_{T L}, v_{T T}\right)$ are presented

$$
v_{L T}=V_{f} v_{l t f}+V_{m} v_{m}
$$




$$
\begin{gathered}
v_{T L}=v_{L T} \frac{E_{T}}{E_{L}} \\
v_{T T}=V_{f} v_{t t f}+V_{m}\left(2 v_{m}-v_{T L}\right)
\end{gathered}
$$

in which $v_{l t f}$ and $v_{t t f}$ are the Poisson's ratios of the fibers in $l-t$ and $t-t$ directions. Poisson's ratio of the isotropic matrix is indicated with $v_{m}$. In the following equation, density of the composite $(\rho)$ is calculated where $\rho_{f}$ and $\rho_{m}$ signify density of the fibers and matrix

$$
\rho=V_{f} \rho_{f}+V_{m} \rho_{m}
$$

According to the fiber failure mode, such as micro buckling or fracture due to shear, there are many different models to estimate the elastic limits of the composite materials in different directions. In this study, the models proposed by Chamis to estimate the tensile and compressive strength of the composite are used for convenience. In this regard, the longitudinal tensile $\left(L_{T}\right)$ and compressive $\left(L_{c}\right)$ strength of the composite are calculated by Eq.(8) and Eq.(9)

$$
\begin{aligned}
& L_{T}=V_{f} L_{t f} \\
& L_{c}=V_{f} L_{c f}
\end{aligned}
$$

in which $L_{t f}$ and $L_{c f}$ are the longitudinal tensile and compressive strength of the fibers. Transverse tensile $\left(T_{T}\right)$ and compressive $\left(T_{C}\right)$ strengths are as follows

$$
\begin{aligned}
& T_{T}=T_{t m}\left[1-\left(\sqrt{V_{f}}-V_{f}\right)\left(1-\frac{E_{m}}{E_{t f}}\right)\right] \\
& T_{C}=T_{c m}\left[1-\left(\sqrt{V_{f}}-V_{f}\right)\left(1-\frac{E_{m}}{E_{t f}}\right)\right]
\end{aligned}
$$

in Eqs.(10)-(11), terms that are titled as $T_{t m}$ and $T_{c m}$ express tensile and compressive strengths of the matrix material.

\section{Analytical Solution}

In order to define elastic relations, cylindrical polar coordinate system $(r, \theta, z)$ is exploited. Radial, tangential, and axial elastic strains are given below

$$
\varepsilon_{r}=\frac{d u_{r}(r)}{d r}, \varepsilon_{\theta}=\frac{u_{r}(r)}{r}, \varepsilon_{z}=0
$$

where $u_{r}(r)$ is the radial displacement. Due to the axial symmetry of the cylinders, displacement is function of $r$ only. In addition, the axial strain is equal to zero since the ends of the cylinders are considered as fixed. As can be noticed, strain-displacement relation is defined at Eq.(12), and the composite material properties are calculated in the above section. Under these conditions, strain-stress relation can be determined adequately. Recalling from the definition of the problem that the fibers are unidirectionally aligned in the circumferential direction, and the remaining radial and axial directions of the cylinders are taken as transverse. These considerations yield to the following compliance matrix, which portrays the strain-stress relation 


$$
\left[\begin{array}{l}
\varepsilon_{r} \\
\varepsilon_{\theta} \\
\varepsilon_{z}
\end{array}\right]=\left[\begin{array}{rrr}
\frac{1}{E_{T}} & -\frac{v_{L T}}{E_{L}} & -\frac{v_{T T}}{E_{T}} \\
-\frac{v_{T L}}{E_{T}} & \frac{1}{E_{L}} & -\frac{v_{T L}}{E_{T}} \\
-\frac{v_{T T}}{E_{T}} & -\frac{v_{L T}}{E_{L}} & \frac{1}{E_{T}}
\end{array}\right]\left[\begin{array}{l}
\sigma_{r} \\
\sigma_{\theta} \\
\sigma_{z}
\end{array}\right]
$$

in which $\sigma_{r}, \sigma_{\theta}$ and $\sigma_{z}$ express radial, tangential, and axial stresses respectively. Correspondingly, elastic stress-strain relation is obtained with the indicated below stiffness matrix

$$
\begin{gathered}
{\left[\begin{array}{c}
\sigma_{r} \\
\sigma_{\theta} \\
\sigma_{z}
\end{array}\right]=\left[\begin{array}{ccc}
\frac{1-v_{L T} v_{T L}}{E_{L} E_{T} \Delta} & \frac{v_{L T}\left(1+v_{T T}\right)}{E_{L} E_{T} \Delta} & \frac{v_{T T}+v_{L T} v_{T L}}{E_{L} E_{T} \Delta} \\
\frac{v_{T L}\left(1+v_{T T}\right)}{E_{T}^{2} \Delta} & \frac{1-v_{T T}^{2}}{E_{T}^{2} \Delta} & \frac{v_{T L}\left(1+v_{T T}\right)}{E_{T}^{2} \Delta} \\
\frac{v_{T T}+v_{L T} v_{T L}}{E_{L} E_{T} \Delta} & \frac{v_{T L}\left(1+v_{T T}\right)}{E_{L} E_{T} \Delta} & \frac{1-v_{L T} v_{T L}}{E_{L} E_{T} \Delta}
\end{array}\right]\left[\begin{array}{c}
\varepsilon_{r} \\
\varepsilon_{\theta} \\
\varepsilon_{z}
\end{array}\right],} \\
\Delta=\frac{\left(1+v_{T T}\right)\left(1-v_{T T}-2 v_{A T} v_{T A}\right)}{E_{A} E_{T}^{2}}
\end{gathered}
$$

It is to be remarked that both compliance and stiffness matrices are symmetrical and obey Hook's law. In the following, the compatibility condition for the elastic problem is given

$$
r \frac{d \varepsilon_{\theta}}{d r}+\varepsilon_{\theta}-\varepsilon_{r}=0
$$

The compatibility condition is fulfilled by substituting radial and tangential strains given in Eq.(12) to the above equation. The non-trivial equilibrium equation for this problem is of the form

$$
\frac{d \sigma_{r}}{d r}+\frac{1}{r}\left(\sigma_{r}-\sigma_{\theta}\right)+\rho \omega^{2} r=0
$$

where $\omega$ is the angular velocity. To be able to solve Eq.(16), initially, directional strain terms presented in Eq.(12) should be substituted into Eq.(14). Followingly, elastic stresses in Eq.(14) are substituted to Eq.(16). After several algebraic procedures, a non-homogeneous Cauchy Euler type differential equation is obtained

$$
r^{2} \frac{d u_{r}^{2}}{d r^{2}}+r \frac{u_{r}}{d r}-\frac{s_{22}}{s_{11}} u_{r}=-\frac{\rho \omega^{2}}{s_{11}} r^{3}
$$

$s_{i j}(i, j=1,2,3)$ terms used above are the terms of the stiffness matrix given in Eq.(14). In order to solve the homogeneous part of the above differential equation, in other words, the lefthand side of Eq.(17), we may propose a solution as $u_{r}=r^{\lambda}$ in which $\lambda$ is an unknown constant. Successively, $u_{r}^{\prime}=\lambda r^{\lambda-1}$ and $u_{r}^{\prime \prime}=\lambda(\lambda-1) r^{\lambda-2}$ where the superscript (') denotes the derivative. By substituting $u_{r}, u_{r}^{\prime}$ and $u_{r}^{\prime \prime}$ into Eq.(17) and setting the right-hand side of the equation as zero, we obtain the homogeneous solution of Eq.(17) which is named $u_{h}(r)$ 


$$
u_{h}(r)=C_{1} r^{-\alpha_{1}}+C_{2} r^{\alpha_{1}}, \text { and } \alpha_{1}=\sqrt{\frac{s_{22}}{s_{11}}}=\sqrt{\frac{E_{L}\left(1-v_{T T}{ }^{2}\right)}{E_{T}\left(1-v_{L T} v_{T L}\right)}}
$$

In the above equation, $C_{1}$ and $C_{2}$ are the arbitrary constants. The next step is finding the particular solution $\left(u_{P}(r)\right)$ of Eq.(17). The method of variation of parameters is employed for this purpose.

$$
u_{P}(r)=Q_{1}(r) u_{1}(r)+Q_{2}(r) u_{2}(r)
$$

$u_{1}$ and $u_{2}$ are the two homogeneous solutions of Eq.(17) which are equal to $r^{-\alpha_{1}}$ and $r^{\alpha_{1}}$ respectively

$$
Q_{1}(r)=-\int \frac{P(r) u_{2}}{u_{1} u_{2}^{\prime}-u_{1}^{\prime} u_{2}} d r, \quad Q_{2}(r)=\int \frac{P(r) u_{1}}{u_{1} u_{2}^{\prime}-u_{1}^{\prime} u_{2}} d r
$$

in which $P(r)=-\left(\rho \omega^{2} / s_{11}\right) r . P(r)$ is simply found by dividing Eq.(17) with $r^{2}$ and taking the right-hand side of the remaining equation. To be able to utilize the method of variation of parameters, the coefficient of the highest order derivative must be 1 . The particular solution of Eq.(17) takes the below form after these mathematical operations

$$
u_{P}(r)=\alpha_{2} r^{3}, \alpha_{2}=\frac{\rho \omega^{2}}{s_{22}-9 s_{11}}=\frac{\Delta E_{L} E_{T}^{2} \rho \omega^{2}}{E_{L}\left(1-v_{T T}{ }^{2}\right)-9 E_{T}\left(1-v_{L T} v_{T L}\right)}
$$

Finally, radial displacements of the composite cylinders are achieved by adding the homogeneous and particular solutions stated in Eq.(18) and Eq.(21)

$$
u_{r}(r)=C_{1} r^{-\alpha_{1}}+C_{2} r^{\alpha_{1}}+\alpha_{2} r^{3}
$$

Directional elastic strains can be written of the form by applying Eq.(12) to the above equation

$$
\begin{gathered}
\varepsilon_{r}(r)=-C_{1} \alpha_{1} r^{-\alpha_{1}-1}+C_{2} \alpha_{1} r^{\alpha_{1}-1}+3 \alpha_{2} r^{2} \\
\varepsilon_{\theta}(r)=C_{1} r^{-\alpha_{1}-1}+C_{2} r^{\alpha_{1}-1}+\alpha_{2} r^{2}
\end{gathered}
$$

It should be reminded that due to the fixed ends of the geometry, the axial strain is equal to zero. Subsequently, if Eq.(23) and Eq.(24) are implemented to Eq.(14), directional elastic stresses become

$$
\begin{aligned}
& \sigma_{r}(r)=-C_{1} r^{-\alpha_{1}-1}\left(\alpha_{1} s_{11}-s_{12}\right)+C_{2} r^{\alpha_{1}-1}\left(\alpha_{1} s_{11}+s_{12}\right)+\alpha_{2} r^{2}\left(3 s_{11}+s_{12}\right) \\
& \sigma_{\theta}(r)=-C_{1} r^{-\alpha_{1}-1}\left(\alpha_{1} s_{21}-s_{22}\right)+C_{2} r^{\alpha_{1}-1}\left(\alpha_{1} s_{21}+s_{22}\right)+\alpha_{2} r^{2}\left(3 s_{21}+s_{22}\right) \\
& \sigma_{z}(r)=-C_{1} r^{-\alpha_{1}-1}\left(\alpha_{1} s_{31}-s_{32}\right)+C_{2} r^{\alpha_{1}-1}\left(\alpha_{1} s_{31}+s_{32}\right)+\alpha_{2} r^{2}\left(3 s_{31}+s_{32}\right)
\end{aligned}
$$

in which $s_{i j}$ terms, once again, express the components of the stiffness matrix in Eq.(14). In the case of rotating cylinders with central holes, $C_{1}$ and $C_{2}$ are found by the following boundary conditions

$$
\sigma_{r}(a)=0, \quad \sigma_{r}(b)=0
$$


in which $a$ and $b$ present inner and outer radii of the cylinders. If the boundary conditions are exerted to Eq.(25), arbitrary constants can be carried out

$$
\begin{gathered}
C_{1}=\frac{\alpha_{2}\left(a^{2 \alpha_{1}} b^{3+\alpha_{1}}-a^{3+\alpha_{1}} b^{2 \alpha_{1}}\right)\left(3 s_{11}+s_{12}\right)}{\left(a^{2 \alpha_{1}}-b^{2 \alpha_{1}}\right)\left(\alpha_{1} s_{11}-s_{12}\right)} \\
C_{2}=-\frac{\alpha_{2}\left(a^{3+\alpha_{1}}-b^{3+\alpha_{1}}\right)\left(3 s_{11}+s_{12}\right)}{\left(a^{2 \alpha_{1}}-b^{2 \alpha_{1}}\right)\left(\alpha_{1} s_{11}+s_{12}\right)}
\end{gathered}
$$

As previously stated, Hoffman yield criterion is employed to calculate the elastic limits of the cylinders. The general form of the corresponding criterion in principal directions is

$$
\begin{aligned}
& \frac{1}{2}\left(-\frac{1}{X_{T} X_{C}}+\frac{1}{Y_{T} Y_{C}}+\frac{1}{Z_{T} Z_{C}}\right)\left(\sigma_{\theta}-\sigma_{z}\right)^{2}+\frac{1}{2}\left(\frac{1}{X_{T} X_{C}}-\frac{1}{Y_{T} Y_{C}}+\frac{1}{Z_{T} Z_{C}}\right)\left(\sigma_{z}-\sigma_{r}\right)^{2}+ \\
& \frac{1}{2}\left(\frac{1}{X_{T} X_{C}}+\frac{1}{Y_{T} Y_{C}}-\frac{1}{Z_{T} Z_{C}}\right)\left(\sigma_{r}-\sigma_{\theta}\right)^{2}+\left(\frac{1}{X_{T}}-\frac{1}{X_{C}}\right) \sigma_{r}+\left(\frac{1}{Y_{T}}-\frac{1}{Y_{C}}\right) \sigma_{\theta}+ \\
& \quad\left(\frac{1}{Z_{T}}-\frac{1}{Z_{C}}\right) \sigma_{z} \leq 1
\end{aligned}
$$

at Eq.(31), $X, Y, Z$ express the yield strength of the material at the corresponding direction, and the subscripts $T$ and $C$ clarify either the load is tensile or compressive. In-between equations Eqs.(8)-(11), composite material strengths have previously been introduced. By operating Eq.(8) to Eq.(11) with Eq.(31), Hoffman yield criteria can be adapted to this case

$$
\begin{gathered}
\sigma_{Y}(r)=\frac{1}{2 L_{T} L_{C}}\left[\left(\sigma_{\theta}-\sigma_{z}\right)^{2}+\left(\sigma_{r}-\sigma_{\theta}\right)^{2}\right]+\frac{1}{2}\left(\frac{2}{T_{T} T_{C}}-\frac{1}{L_{T} L_{C}}\right)\left(\sigma_{z}-\sigma_{r}\right)^{2} \\
+\left(\frac{1}{T_{T}}-\frac{1}{T_{C}}\right)\left(\sigma_{r}+\sigma_{z}\right)+\left(\frac{1}{L_{T}}-\frac{1}{L_{C}}\right) \sigma_{\theta} \leq 1
\end{gathered}
$$

Eq.(32) is the corresponding yield criteria, which is named $\sigma_{Y}(r)$ and is going to be used to find the elastic limit angular velocity of the cylinders. If the value of the obtained $\omega$ is exceeded, then the elastic region gets exited. In other words, from Eq.(12) to this point, all elastic equations are valid as long as $\sigma_{Y}(r) \leq 1$. Thus, limit angular velocity values are obtained when $\sigma_{Y}(r)=1$.

\section{Numerical Examples}

After analytical modeling, to exemplify numerical results, dimensions of the composite cylinders and the properties of the cylinder material are assigned. In this regard, to understand how the wall thickness of the cylinders changes the distribution of the elastic stresses, outer radii $(b)$ of the cylinders are kept constant as $0.1 \mathrm{~m}$, and various inner radii $(a)$ values $(0.02$ $\mathrm{m}, 0.05 \mathrm{~m}, 0.08 \mathrm{~m})$ are taken. So that the inner/outer radius ratio $(a / b)$ becomes $0.2,0.5$ and 0.8 respectively. As the fiber reinforced composite, graphite/epoxy is used. Properties of the epoxy and transversely isotropic graphite fibers are tabulated below

Table 1. Mechanical Properties of the Epoxy and Graphite Fibers [25]

\begin{tabular}{cccccccccccc}
\hline$E_{l f}$ & $E_{t f}$ & $E_{m}$ & $v_{l t f}$ & $v_{t t f}$ & $v_{m}$ & $\rho_{f}$ & $\rho_{m}$ & $L_{t f}$ & $L_{c f}$ & $T_{t m}$ & $T_{c m}$ \\
\hline$(G P a)$ & $(G P a)$ & $(G P a)$ & $(-)$ & $(-)$ & $(-)$ & $\left(\frac{\mathrm{kg}}{\mathrm{m}^{3}}\right)$ & $\left(\frac{\mathrm{kg}}{\mathrm{m}^{3}}\right)$ & $(\mathrm{MPa})$ & $(\mathrm{MPa})$ & $(\mathrm{MPa})$ & $(\mathrm{MPa})$ \\
\hline 230 & 22 & 3.4 & 0.30 & 0.35 & 0.30 & 1800 & 1200 & 2067 & 1999 & 72 & 102 \\
\hline
\end{tabular}


Material properties are obtained for three different fiber volume fraction values $\left(V_{f}=0.20\right.$, $0.50,0.80$ ) via operating Chamis method, which is given from (1) to (11), with the properties in Table 1. Accordingly, one can investigate the influences of $V_{f}$ on the elastic stress and displacement fields. At this stage, variables are transformed to their normalized forms which are expressed with overbars. Hence, radial coordinate is $\bar{r}=r / b$. Directional stresses take the following forms $\bar{\sigma}_{j}(r)=\sigma_{j}(r) / \sigma_{o}$ where $j=r, \theta, z$ and the yield stress is $\bar{\sigma}_{Y}(r)=\sigma_{Y}(r)$. Normalized angular velocity can be written as $\bar{\omega}=\omega b \sqrt{\rho_{o} / \sigma_{o}}$. Non-dimensional radial displacement is transformed to $\bar{u}_{r}(r)=u_{r}(r) E_{o} / \sigma_{o} b$. Lastly, arbitrary constant are of the following $\bar{C}_{1}=C_{1} / b^{1+\alpha_{1}}$ and $\bar{C}_{2}=C_{2} / b^{1-\alpha_{1}}$. In the normalization procedure $\sigma_{o}, E_{o}$ and $\rho_{o}$ are introduced. These constants are as follows

$$
\sigma_{o}=\frac{1}{4} \sqrt{\left(\frac{L_{t f}+L_{c f}+T_{t m}+T_{c m}}{4}\right)^{2}}, E_{o}=\frac{1}{3} \sqrt{\left(\frac{E_{l f}+E_{t f}+E_{m}}{3}\right)^{2}} \text { and } \rho_{o}=\frac{\rho_{f}+\rho_{m}}{2}
$$

After the boundary conditions stated in Eq.(29) and Eq.(30) are employed to stresses and displacements, elastic limit angular velocity and the beginning of the yielding are calculated by the use of Eq.(32). The results obtained for various $a / b$ and $V_{f}$ are exhibited in Table 2 . It is to be noted that the position of the yielding is denoted with $\bar{r}_{Y}$ in Table 2

Table 2. Calculated dimensionless arbitrary constants, elastic limit angular velocities and position of the yielding for various $V_{f}$ and $a / b$

\begin{tabular}{l|r|rrr}
\hline \multicolumn{2}{c}{} & \multicolumn{1}{c}{$V_{f}=0.20$} & $V_{f}=0.50$ & \multicolumn{1}{c}{$V_{f}=0.80$} \\
\hline & $\bar{C}_{1}$ & $6.67953 \times 10^{-6}$ & $7.69944 \times 10^{-7}$ & $4.93349 \times 10^{-7}$ \\
$a / b=0.2$ & $\bar{C}_{2}$ & 0.0341764 & -0.0388193 & -0.0243708 \\
& $\bar{r}_{Y}$ & 0.618322 & 0.645025 & 0.634944 \\
& $\bar{\omega}$ & 1.56408 & 1.8232 & 1.84966 \\
\hline \multirow{3}{*}{$a / b=0.5$} & $\bar{C}_{1}$ & 0.000354862 & 0.000141208 & 0.000103404 \\
& $\bar{C}_{2}$ & 0.0316035 & -0.0445137 & -0.0298333 \\
& $\bar{r}_{Y}$ & 0.5 & 0.720314 & 0.722186 \\
& $\bar{\omega}$ & 1.49828 & 1.95469 & 2.04922 \\
\hline & $\bar{C}_{1}$ & 0.001605 & 0.00120456 & 0.00109806 \\
& $\bar{C}_{2}$ & 0.0263474 & -0.0435268 & -0.0351421 \\
& $\bar{r}_{Y}$ & 0.8 & 0.8 & 0.8 \\
& $\bar{\omega}$ & 1.34404 & 1.95086 & 2.24789 \\
\hline
\end{tabular}

It is depicted in the results in Table 2 that elastic limit angular velocity increases with higher fiber volume fraction. In other words, when the fiber volume fraction elevates, cylinders begin yielding at higher strengths. Whereas, it is hard to make a clear statement for the influence of the $a / b$ ratio. When $V_{f}=0.20$ and $a / b$ ratio is increasing from 0.20 to 0.80 , calculated elastic limit angular velocities decreas. On the other hand, when $V_{f}=0.80$ and $a / b$ ratio rises, composite cylinders tend to fail at higher $\bar{\omega}$. Thus, one can conclude that the wall thickness of the composite cylinders should be treated carefully to get optimum results. In order to understand this phenomenon, Figure 2 is illustrated below. According to the plotting, when fiber volume fraction in the composite is low $\left(V_{f}=0.20\right)$, thick-walled cylinders $(a / b=0.20)$ are prone to yield at greater $\bar{\omega}$. However, as $V_{f}$ increases in the composite, cylinders that have thinner wallthickness $(a / b=0.80)$ start plastic flow at higher $\bar{\omega}$. 


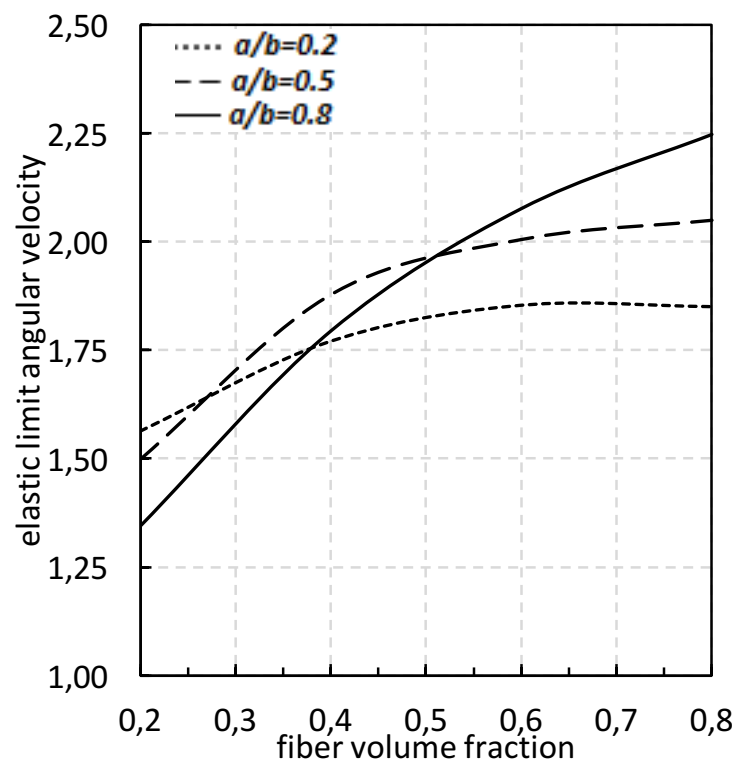

Fig. 2. Distribution of the elastic limit angular velocity for the cylinders having different $a / b$ and $V_{f}$

In the following figures, corresponding elastic stress and displacement fields are presented. As can be noticed in Figure 3 (a), (b), and (c), when $a / b=0.2$, plastic flow commences in the middle of the cylinders. On the other hand, as the thickness of the cylinder walls gets slender, yielding begins at the inner radii. For instance, all cylinders begin yielding at $r=a$ when $a / b=0.8$. The position of the yielding $\left(\bar{r}_{Y}\right)$ is calculated when $\bar{\sigma}_{Y}(r)=1$, and the exact position of $\bar{r}_{Y}$ is given in Table 2
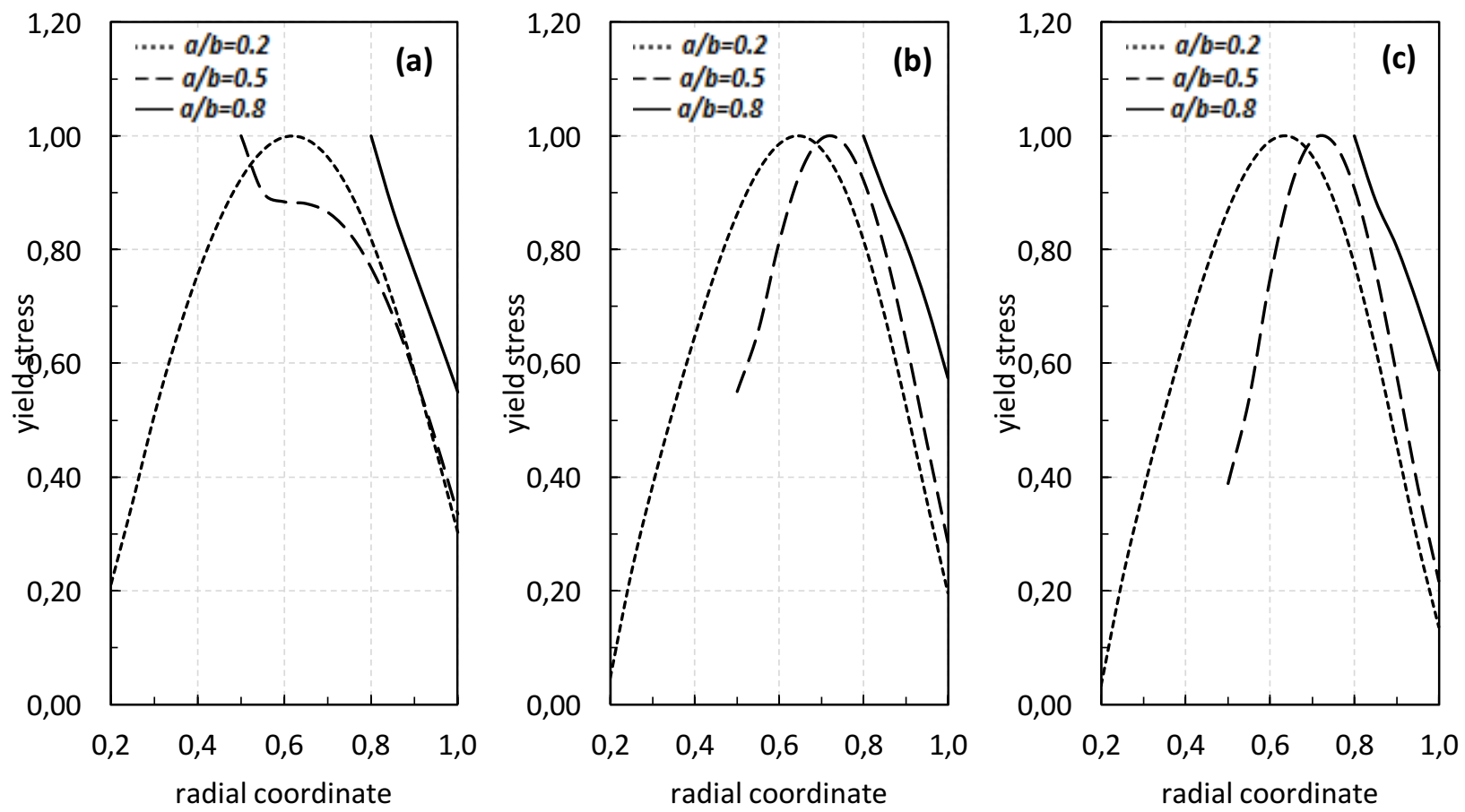

Fig. 3. Variation of the dimensionless yield stress along $\bar{r}$ for various $a / b$ ratios where (a) $V_{f}=0.20$,

(b) $V_{f}=0.50$ and (c) $V_{f}=0.80$ 
In the subsequent set of figures, non-dimensional elastic limit radial stresses are presented. It is observed from Figure 4 (a), (b), and (c) that radial stresses increase as $V_{f}$ enlarges for the cylinders with the same $a / b$ ratio. The graphs below also reveal that the cylinders with higher wall thicknesses $(a / b=0.2)$ have higher radial stress compared to the lower ones $(a / b=0.8)$. In the ensuing plotting, tangential stresses are exhibited
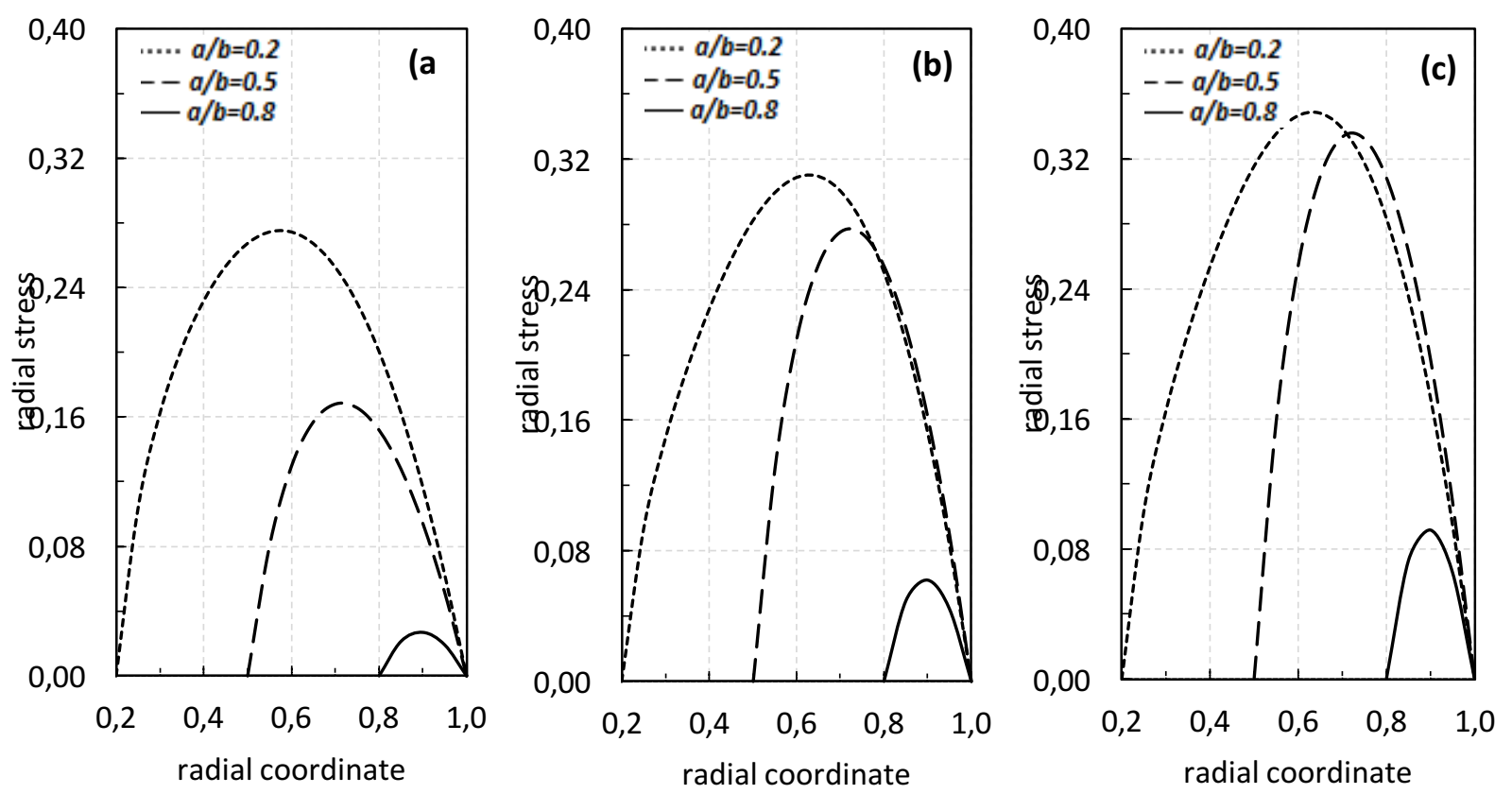

Fig. 4. Variation of the dimensionless radial stress along $\bar{r}$ for various $a / b$ ratios where (a) $V_{f}=0.20$,

(b) $V_{f}=0.50$ and (c) $V_{f}=0.80$


Fig. 5. Variation of the dimensionless tangential stress along $\bar{r}$ for various $a / b$ ratios where (a) $V_{f}=0.20$, (b) $V_{f}=0.50$ and (c) $V_{f}=0.80$ 
According to Figure 5 (a), (b), and (c), normalized tangential stresses enlarge, when $V_{f}$ amplifies for cylinders with the same $a / b$ ratio. On the other hand, while the radial stresses are high in the thick-walled cylinders $(a / b=0.2)$, tangential stress components are high in the thinner ones $(a / b=0.8)$. Another important issue is that the magnitudes of the elastic limit tangential stresses are significantly higher than the radial stresses. In the next figure, axial stresses are presented in Figure 6 (a), (b), and (c). Fiber volume fraction slightly influences the axial stresses when $a / b=0.2$. Conversely, as the wall thickness gets smaller, the effect of $V_{f}$ becomes more apparent and the profiles of the axial stress components tend to skew to the outer radii of the cylinders.
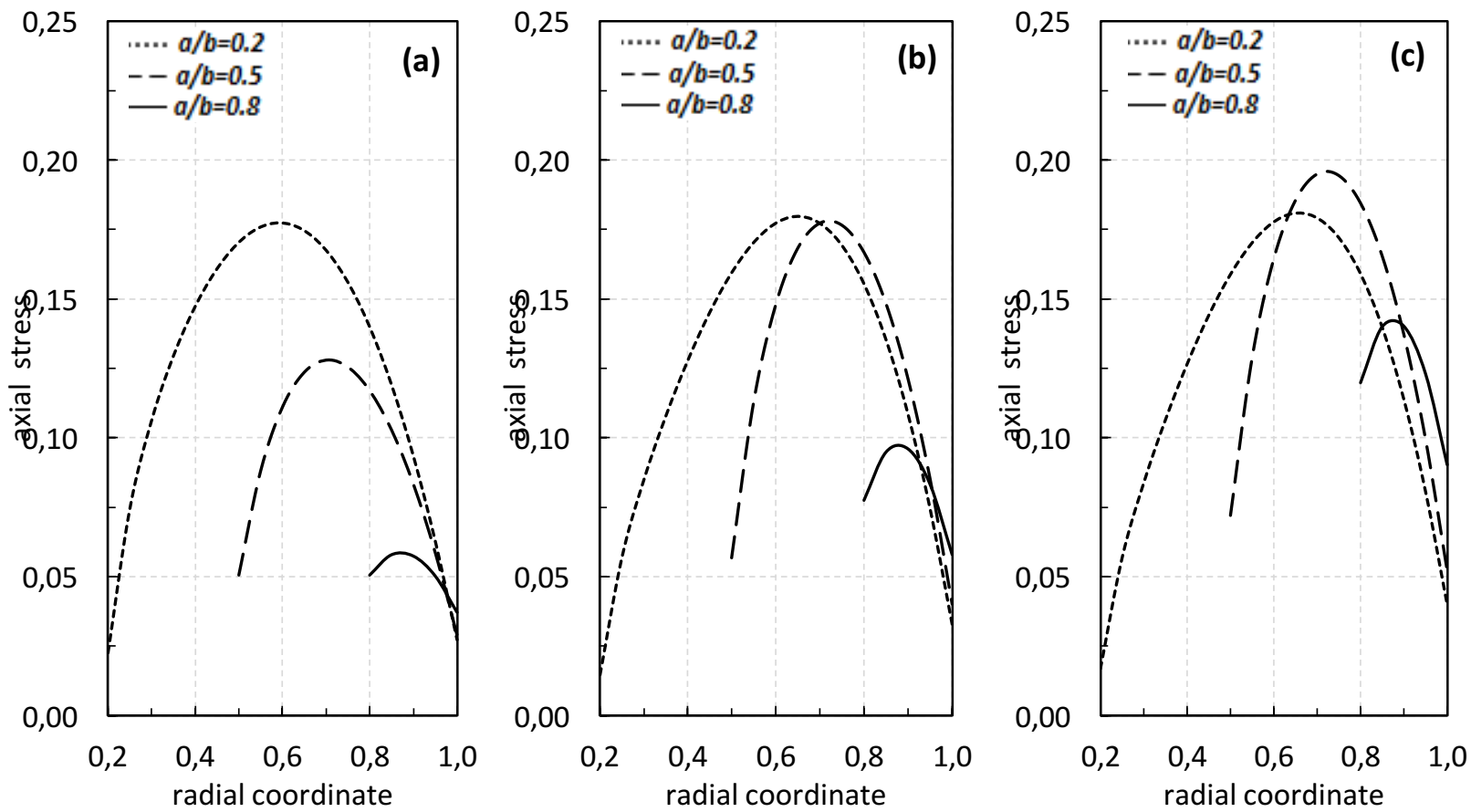

Fig. 6. Variation of the dimensionless axial stress along $\bar{r}$ for various $a / b$ ratios where (a) $V_{f}=0.20$,

(b) $V_{f}=0.50$ and (c) $V_{f}=0.80$

In the final figure demonstrated below, normalized radial displacements are portrayed. In accordance with Figure 7 (a), (b), and (c), radial displacements of the cylinders sharing the same $a / b$ reduce as $V_{f}$ goes up. For the cylinders having the same $V_{f}$, radial displacements are highly affected by $a / b$. When the cylinders are having narrower walls, the magnitudes of the radial displacements are at high levels. However, the radial displacement differences of the inner and outer radii of the cylinders become greater in the thick-walled ones. Another remark that should be noted is that the distribution profiles of the radial displacements and tangential stresses are markedly analogous. The resemblance can be noticed by comparing Figures 5 and 7. 

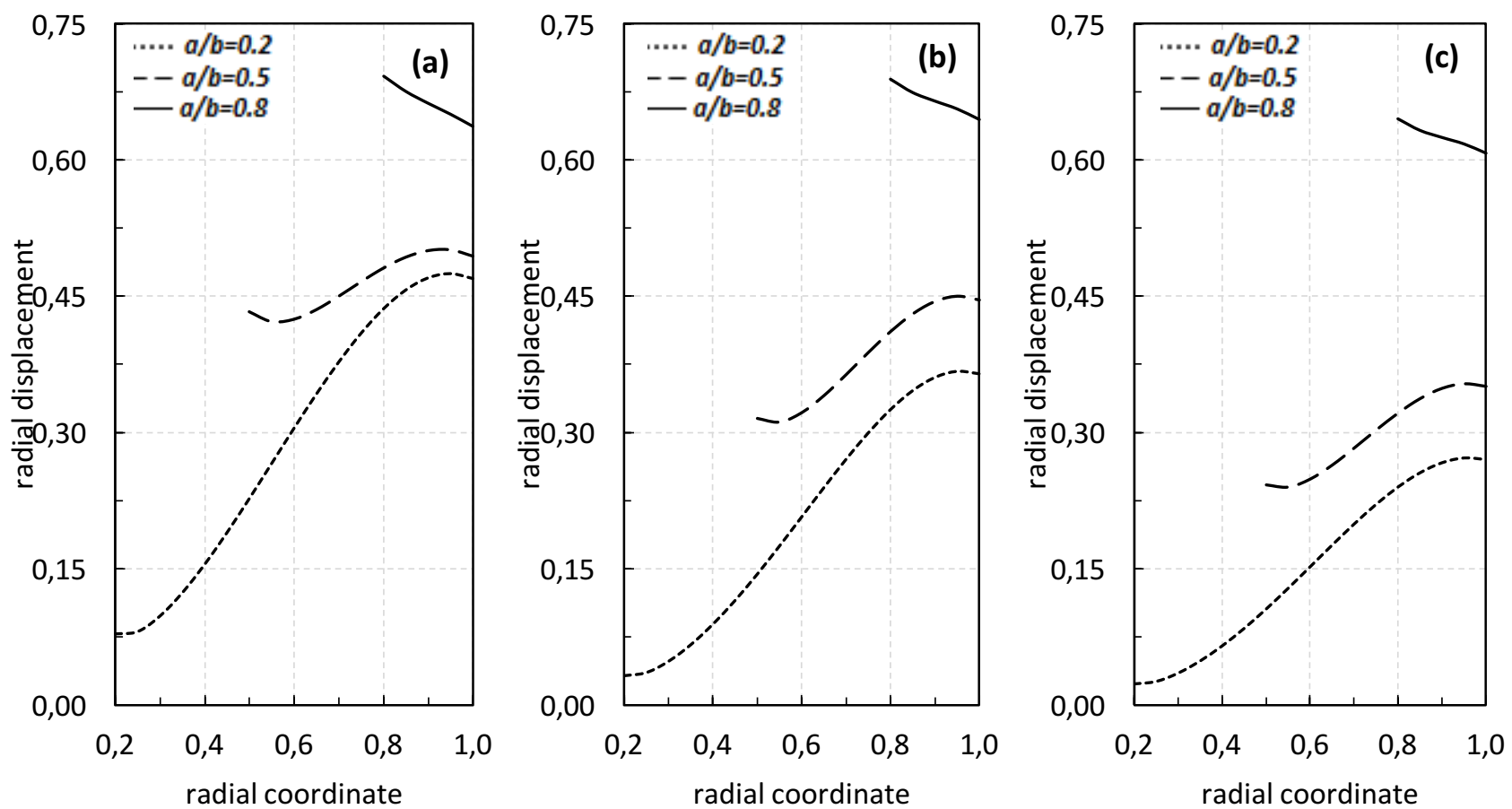

Fig. 7. Variation of the dimensionless radial displacement along $\bar{r}$ for various $a / b$ ratios where (a) $V_{f}=0.20$, (b) $V_{f}=0.50$ and (c) $V_{f}=0.80$

\section{Conclusion}

In this study, the rotating fiber reinforced composite cylinders subjected to free-free boundary conditions are studied by employing analytical methods. The elastic stress and displacement fields are investigated for numerous wall thickness $(a / b)$ and fiber volume $\left(V_{f}\right)$ ratios. It has been seen that these fields are immensely altered by $a / b$ and $V_{f}$. Hoffman yield criterion is adapted to the problem and elastic limits are obtained accordingly. One can conclude from the calculated results that as $V_{f}$ in the composite increases, yielding initiates at higher angular velocities. Whereas, $a / b$ ratio should be utilized cautiously. If the wall thickness ratio is not selected properly, yielding in the cylinders may occur at lower angular velocities. The commencement of the plastic flow varies according to the assigned $a / b$ and $V_{f}$. When the cylinder walls are thick, yielding tends to begin in the middle of the cylinder walls. However, as the wall thickness becomes smaller, yielding starts at the inner radii of the cylinders. Also, according to the elastic limit stress and displacement fields that we get, magnitudes of radial and axial stresses are considerably small when compared to tangential ones, and as the cylinder walls get thinner tangential stress components majorly rise.

\section{References}

[1] Dai, T., Dai, H. L., Investigation of mechanical behavior for a rotating fgm circular disk with a variable angular speed, Journal of Mechanical Science and Technology, 29(9), 3779-3787, 2015.

[2] Eraslan, A. N., Arslan, E., Analytical and numerical solutions to rotating fgm disk, Journal of Multidisciplinary Engineering Science and Technology, 2(10), 2843-2850, 2015. 
[3] Zheng, Y., Bahaloo, H., Mousanezhad, D., Mahdi, E., Vaziri, A., Nayeb-Hashemi, H., Stress analysis in functionally graded rotating disks with non-uniform thickness and variable angular velocity, International Journal of Mechanical Sciences, 119, 283-293, 2016.

[4] Çallığlu, H., Bektaş, N. B., Sayer, M., Stress analysis of functionally graded rotating disc: analytical and numerical solutions, Acta Mechanica Sinica, 27(6), 950-955, 2011.

[5] Horgan, C. O., Chan, A. M., The stress response of functionally graded isotropic linearly elastic rotating disks, Journal of Elasticity, 55(3), 219-230, 1999.

[6] Y1ldırım, V., Effects of inhomogeneity and thickness parameters on the elastic response of a pressurized hyperbolic annulus/disc made of functionally graded material, International Journal of Engineering \& Applied Sciences (IJEAS), 9(3), 36-50, 2017.

[7] Dui, G., Zhou, D., Solutions for behavior of a functionally graded thick-walled tube subjected to mechanical and thermal loads, International Journal of Mechanical Sciences, 98, 70-79, 2015.

[8] Dui, G., Zhang, J., An elasticity solution for functionally graded thick-walled tube subjected to internal pressure, International Journal of Mechanical Sciences, 89, 344-349, 2014.

[9] Tütütncü, N., Stresses in thick-walled FGM cylinders with exponentially-varying properties, Engineering Structures, 29(9), 2032-2035, 2007.

[10] Nejad, M. Z., Rahimi, G. H., Deformations and stresses in rotating fgm pressurized thick hollow cylinder under thermal load, Scientific Research and Essays, 4(3), 131-140, 2009.

[11] Nie, G. J., Zhong, Z., Batra, R. C., Material tailoring for orthotropic elastic rotating disks, Composites Science and Technology, 71(3), 406-414, 2011.

[12] Genta, G., Gola, M., The stress distribution in orthotropic rotating disks, Journal of Applied Mechanics, 48(3), 559-562, 1981.

[13] Çallığlu, H., Stress analysis of an orthotropic rotating disc under thermal loading, Journal of Reinforced Plastics and Composites, 23(17), 1859-1867, 2004.

[14] Eraslan, A. N., Kaya, Y., Varl1, E., Analytical solutions to orthotropic variable thickness disk problems, Pamukkale University Journal of Engineering Sciences, 22(1), 24-30, 2016.

[15] El-Naggar, A. M., Abd-Alla, A. M., Fahmy, M.A., Ahmed, S. M., Thermal stresses in a rotating non-homogeneous orthotropic hollow cylinder, Heat and Mass Transfer, 39(1), 41-46, 2002.

[16] Haojiang, D., Huiming, W., Weiqiu, C., Transient thermal stresses in an orthotropic hollow cylinder for axisymmetric problems, Acta Mechanica Sinica, 20(5), 477-483, 2004.

[17] El-Naggar, A. M., Abd-Alla, A. M., Ahmed, S. M., On the rotation of a non-homogeneous composite infinite cylinder of orthotropic material, Applied Mathematics and Computation, 69(2-3), 147-157, 1995. 
[18] Abd-Alla, A. M., Mahmoud, S. R., Shehri, N. A., Effect of the rotation on a nonhomogeneous infinite cylinder of orthotropic material, Applied Mathematics and Computation, 217(22), 8914-8922, 2011.

[19] Zheng, Y., Bahaloo, H., Mousanezhad, D., Vaziri, A., Displacement and stress fields in a functionally graded fiber-reinforced rotating disk with nonuniform thickness and variable angular velocity, Journal of Engineering Materials and Technology, 139(3), 031010-10, 2017.

[20] Zenkour, A. M., Allam, M. N. M., On the rotating fiber-reinforced viscoelastic composite solid and annular disks of variable thickness, International Journal for Computational Methods in Engineering Science and Mechanics, 7(1), 21-31, 2006.

[21] Tahani, M., Nosier, A., Zebarjad, S. M., Deformation and stress analysis of circumferentially fiber-reinforced composite disks, International journal of solids and structures, 42(9-10), 2741-2754, 2005.

[22] Hoffman, O., The brittle strength of orthotropic materials, Journal of Composite Materials, 1(2), pp. 200-206, 1967.

[23] Chamis, C. C., Mechanics of composite materials: past, present, and future, Journal of Composites, Technology and Research, 11(1), 3-14, 1989.

[24] Chamis, C. C., Simplified composite micromechanics equations for strength, fracture toughness, impact resistance and environmental effects, Nasa Technical Memorandum (NASA-E-2154), Clevland, OH, 1984.

[25] Kaw, A. K., Mechanics of Composite Materials, CRC Press; $2^{\text {nd }}$ edition, 2002. 